\title{
FAS and FASL genetic polymorphisms impact on clinical outcome of malignant pleural mesothelioma
}

\author{
Manal El-Hamamsy' \\ Ramy R Ghali² \\ Amr S Saad ${ }^{2}$ \\ Sara M Shaheen' \\ Ahmed M Salem' \\ 'Department of Clinical Pharmacy, \\ Faculty of Pharmacy, Ain Shams \\ University, Cairo, Egypt; ${ }^{2}$ Department \\ of Clinical Oncology, Faculty of \\ Medicine, Ain Shams University, \\ Cairo, Egypt
}

This article was published in the following Dove Press journal:

OncoTargets and Therapy

7 November 2016

Number of times this article has been viewed

Background: FAS-670 A $>$ G (rs1800682) and FASL-844 C $>$ T (rs763110) polymorphisms have been previously correlated with clinical outcome of non-small cell lung cancer (NSCLC) and breast and bladder cancers. We investigated the influence of these polymorphisms on clinical outcome of malignant pleural mesothelioma (MPM) patients.

Patients and methods: In this cohort study (NCT02269878), 68 epithelioid MPM Egyptian patients treated with first-line platinum-based chemotherapy were recruited in the period between April 2014 and May 2015. The genotype analysis was performed using TaqMan ${ }^{\circledR}$ single-nucleotide polymorphism genotyping assay. The association between the selected polymorphisms and response rate, progression-free survival (PFS) and overall survival (OS) at 18 months was evaluated.

Results: The median age of patients was 55 years and $45.6 \%$ of them received platinum in combination with pemetrexed, while $54.4 \%$ received platinum in combination with gemcitabine. FASL-844 CC genotype was more common than expected in early-stage tumor $(P=0.042)$. It was found that there was no association between the investigated polymorphisms and response rate or 18-month OS. However, the PFS rate at 18 months for FASL-844 CC genotype carriers was $45 \%$ versus $10.6 \%$ for FASL-844 CT/TT genotypes carriers (log-rank: $6.2 ; P=0.013$ ). Also, the number of platinum-based cycles and tumor stage were found to be significant variables for PFS by univariate analysis ( $P \leq 0.001$ and $P=0.006$, respectively). Stratified Cox regression showed that the carriers of FASL-844 CT/TT genotypes were still more susceptible to disease progression than carriers of FASL-844 CC genotype (adjusted HR $=3.77,95 \% \mathrm{CI}$ : 1.34-10.62, $P=0.012$ ).

Conclusion: The results of this study suggest that FASL-844 C/T polymorphism could predict PFS in MPM patients receiving platinum-based chemotherapy; therefore, this should be further evaluated as a potential marker for the prediction of clinical outcome in patients with MPM.

Keywords: mesothelioma, platinum, FAS, FAS ligand, genotyping, survival

\section{Introduction}

Malignant mesothelioma is considered one of asbestos-related diseases, which affects the mesothelium of the pleura in the majority of cases. ${ }^{1}$ In Egypt, mesothelioma is considered a dreadful problem due to its increasing incidence and the dismal prognosis where 1- and 2-year survival rates are estimated to be $60 \%$ and $27 \%$, respectively. ${ }^{2}$ Features of mesothelioma associated with poor prognosis include nonepithelioid subtype, advanced age, performance status (PS) $\geq 2$ and advanced tumor stage. ${ }^{3}$

Multimodality therapy comprising surgery, radiotherapy, and chemotherapy offers modest improvement in survival in malignant pleural mesothelioma (MPM) but only
Correspondence: Ahmed M Salem Department of Clinical Pharmacy, Faculty of Pharmacy, Ain Shams University, African Union Organization Street, Abbasiya, Cairo II566, Egypt Tel +20 III I76 680I Fax +2022405 II 07 Email ahmedsalem.salem@pharma.asu. edu.eg 
a limited subset of patients is eligible for this because the majority of patients are diagnosed at advanced stage when the surgical resection of the tumor is impossible. ${ }^{4}$ Hence, platinum-based chemotherapy is considered the main therapeutic strategy for MPM patients. ${ }^{5}$ Cisplatin combined with pemetrexed is the current standard first-line chemotherapy regimen in MPM setting. ${ }^{6}$ An alternative option is the combination of cisplatin and gemcitabine which showed comparable results in phase II trials. ${ }^{7}$ Carboplatin shows similar survival benefits and could replace cisplatin especially for elderly patients who cannot tolerate cisplatin. ${ }^{8}$

FAS ligand (FASL) is a transmembrane protein which binds to its cell receptor, FAS, to initiate apoptosis cascade in cells. ${ }^{9}$ It is proposed that cisplatin exerts its anticancer activity in MPM partly by induction of the FAS pathway. ${ }^{10}$ In addition, studies demonstrate that both gemcitabine and pemetrexed depend on FAS pathway to initiate apoptosis in cancer cells. ${ }^{11,12}$ Hence, this study hypothesizes that expression levels of FAS and FASL may affect anticancer activity of these agents.

FAS-670 A $>$ G and FASL-844 $\mathrm{C}>\mathrm{T}$ are two polymorphisms located in the promoter region of FAS and FASL genes, respectively, and affect their expression levels. ${ }^{13,14}$ Several studies have investigated the association of these polymorphisms with the prediction of clinical outcome and prognosis in non-small cell lung cancer (NSCLC) and breast and bladder cancers. ${ }^{15-19}$ However, the results of these studies are nonconclusive. Moreover, no study has evaluated the effect of these polymorphisms on the clinical outcome in MPM setting.

Consequently, the intention behind this study is to investigate the correlation between FAS-670 $\mathrm{A}>\mathrm{G}$ and FASL-844 C $>$ T polymorphisms, and the clinical outcomes in Egyptian epithelioid MPM patients treated with first-line platinum-based chemotherapy in terms of objective response rate, progression-free survival (PFS), and overall survival (OS) at 18 months.

\section{Materials and methods}

\section{Patients}

Cohort design was adopted in this study. Patients were recruited from April 2014 to May 2015. Patients were included in the study if their clinical, radiological, and immunohistochemical findings were consistent with epithelioid MPM. All patients had PS of 0-2 and had adequate laboratory parameters to start their first-line platinum-based chemotherapy. Also, the cohort included patients who started their first-line platinum-based regimens before the commencement of the study but still were not evaluated for tumor response. The ethics committee of the Faculty of Pharmacy,
Ain Shams University, Cairo, Egypt, approved the initiation or modifications to protocol made after the initiation of the study (approval reference code: Master-No 14). All the participants gave their written informed consent to enroll in the study which was performed according to the Declaration of Helsinki. This study is registered at ClinicalTrials.gov (NCT02269878).

\section{Intervention}

Patients were recruited from the Department of Clinical Oncology and Nuclear Medicine, Ain Shams University (Cairo, Egypt) and El-Nasr hospital for health insurance in Helwan (Cairo, Egypt) which are two leading sites for MPM cases referral and treatment in Egypt. All the patients were subjected to the same standards of practice regarding treatment protocols and outcome assessment. Patients received one of the following protocols: platinum agent, either cisplatin $75 \mathrm{mg} / \mathrm{m}^{2}$ or carboplatin AUC 5, in day 1 plus pemetrexed $500 \mathrm{mg} / \mathrm{m}^{2}$ in day 1 every 3 weeks or platinum agent in day 1 plus gemcitabine $1,250 \mathrm{mg} / \mathrm{m}^{2}$ in day 1 and day 8 every 3 weeks. If pemetrexed was administered, supplementary folic acid and vitamin B12 were also received.

\section{Response and survival assessment}

Computed tomography scans were performed every two cycles, and tumor response to platinum-based chemotherapy was evaluated according to response evaluation criteria in solid tumors version 1.1. ${ }^{20} \mathrm{PFS}$ was calculated from day 1 of the first cycle of platinum-based chemotherapy to the date of documented progression or death due to any cause. OS was calculated from day 1 of starting first cycle of platinum-based chemotherapy to the date of death due to any cause. Follow-up period was determined to be 18 months. The end of follow-up period was May 1, 2016. Patients who completed the follow-up period without dying or showing disease progression were considered censored. Patients who did not complete the follow-up period were censored at the last follow-up visit.

\section{Single-nucleotide polymorphism genotyping assay}

Three milliliters of peripheral blood was collected in collection tubes containing potassium salt of ethylenediaminetetraacetic acid and were stored at $-80^{\circ} \mathrm{C}$ until DNA extraction. DNA was extracted from the blood using QIAamp DNA blood mini kit (Qiagen, Hilden, Germany). Genotyping was performed using TaqMan ${ }^{\circledR}$ SNP genotyping assay (Applied Biosystems $^{\circledR}$, Foster City, CA, USA). Each polymerase chain reaction (PCR) mixture contained $10 \mu \mathrm{L}$ of universal TaqMan master mix, $1 \mu \mathrm{L}(40 \times)$ of diluted TaqMan SNP genotyping 
assay (diluted in 1:1 ratio with purified DNase-free water), 100 ng genomic DNA diluted in DNase-free water to complete the final volume of $20 \mu \mathrm{L}$. PCR conditions were set as follows: initial step at $95^{\circ} \mathrm{C}$ for $10 \mathrm{~min}$ then 50 cycles of each denaturation at $95^{\circ} \mathrm{C}$ for $15 \mathrm{~s}$ and annealing/extension at $60^{\circ} \mathrm{C}$ for $1 \mathrm{~min}$.

\section{Statistical analysis}

Frequencies and percentages were used to summarize categorical data. For continuous data, median and range were used to describe centrality and dispersion, respectively. To test whether the genotype frequencies are in agreement with those expected under Hardy-Weinberg equilibrium, standard chi-square test was performed. To test whether there is an association between the investigated polymorphisms and different clinicopathological variables or response rate, chi-square and Fisher's exact tests were used. For survival data, the Kaplan-Meier method was used to draw survival curves and to estimate the survival rates at 18 months, while log-rank test was used to compare the survival data. All variables that showed statistical significance in univariate analyses were chosen for multivariate analyses. Stratified Cox regression was used to calculate adjusted hazard ratios, 95\% confidence intervals and associated $P$-values. Twosided $P$-value $<0.05$ was considered as a cutoff value for statistical significance. Statistical analysis was performed using IBM SPSS Version 22 (IBM Corporation, Armonk, NY, USA).

\section{Results \\ Patients}

This study included 68 epithelioid MPM patients. Forty four patients $(64.7 \%)$ were recruited from Department of Clinical Oncology and Nuclear Medicine, Ain Shams University and 24 patients (35.3\%) from El-Nasr hospital for health insurance in Helwan. Clinical and treatment characteristics of the cohort are presented in Table 1. Fifty one patients (75\%) received six cycles or more of the first-line platinum-based chemotherapy. Thirty four patients $(50 \%)$ received secondline chemotherapy. Second-line chemotherapy regimens included pemetrexed and platinum combination $(n=19)$, gemcitabine and platinum $(n=8)$, gemcitabine single agent $(n=3)$, and vinorelbine single agent $(n=4)$. Three patients received limited field prophylactic radiotherapy, while nine patients received palliative radiotherapy.

No difference was observed between patients treated with platinum in combination with gemcitabine or patients treated with platinum in combination with pemetrexed in terms of response rate $(P=0.64)$, PFS $(P=0.81)$, or OS $(P=0.47)$.
Table I Clinical and treatment characteristics of cohort of malignant pleural mesothelioma patients receiving platinum-based chemotherapy $(n=68)$

\begin{tabular}{|c|c|c|}
\hline Characteristic & Category & \\
\hline \multirow[t]{2}{*}{ Gender, n (\%) } & Male & $43(36.8)$ \\
\hline & Female & $25(63.2)$ \\
\hline $\begin{array}{l}\text { Age, years, median } \\
\text { (minimum-maximum) }\end{array}$ & & $55(28-75)$ \\
\hline \multirow[t]{2}{*}{ Asbestos exposure, $\mathrm{n}(\%)$} & Exposed & $56(82.4)$ \\
\hline & Unexposed & $12(17.6)$ \\
\hline \multirow[t]{2}{*}{ Smoking history, n (\%) } & Ever-smoker & $40(58.8)$ \\
\hline & Nonsmoker & $28(4 \mid .2)$ \\
\hline \multirow[t]{3}{*}{ IMIG staging, $n(\%)$} & II & $22(32.4)$ \\
\hline & III & $35(51.4)$ \\
\hline & IV & II (I6.2) \\
\hline \multirow[t]{3}{*}{ ECOG performance status, $n(\%)$} & 0 & $10(14.7)$ \\
\hline & 1 & $44(64.7)$ \\
\hline & 2 & $14(20.6)$ \\
\hline \multirow[t]{2}{*}{$\begin{array}{l}\text { First-line chemotherapy } \\
\text { regimen, } n(\%)\end{array}$} & $\begin{array}{l}\text { Platinum in combination } \\
\text { with pemetrexed }\end{array}$ & $31(45.6)$ \\
\hline & $\begin{array}{l}\text { Platinum in combination } \\
\text { with gemcitabine }\end{array}$ & $37(54.4)$ \\
\hline
\end{tabular}

Abbreviations: ECOG, Eastern Cooperative Oncology Group; IMIG, International Mesothelioma Interest Group.

Genotype frequencies of both FASL-844 C $>$ T and FAS$670 \mathrm{~A}>\mathrm{G}$ are presented in Table 2. The genotype frequencies of both the polymorphisms are in concordance with those expected under Hardy-Weinberg equilibrium $(P=0.81$ and $P=0.98$, respectively).

On studying the association between the genotypes of investigated polymorphisms and clinicopathological characteristics, it was found that FASL-844 CC genotype was more common than expected in early-stage tumor while FASL-844 TT genotype was more common than expected in advanced tumor $(P=0.042)$.

\section{Response analysis}

Among the studied cohort, no one achieved complete response, while 20 patients (29.4\%) showed partial response. In addition, this study observed 32 patients (47.1\%) with stable disease and 16 patients $(23.5 \%)$ with progressive disease. No difference was observed between FASL-844 $\mathrm{CC}$ genotype versus FASL-844 CT/TT genotypes $(P=0.98)$ and also between FAS-670 AA genotype versus FAS-670 AG/GG genotypes $(P=0.49)$.

\section{Survival analysis}

At the end of the study, disease progression occurred in 53 patients (77.9\%), seven patients (10.3\%) completed the follow-up period without showing disease progression and eight patients (11.8\%) were lost to follow-up. The PFS rate at 18 months for the entire cohort is $16.4 \%$. Regarding the 
Table 2 Influence of FASL-844 C>T and FAS-670 A > polymorphisms on survival assessed in univariate analysis in patients with malignant pleural mesothelioma treated with platinum-based chemotherapy $(n=68)$

\begin{tabular}{|c|c|c|c|c|c|c|c|c|}
\hline \multirow[t]{2}{*}{ Polymorphism } & \multirow[t]{2}{*}{ Genotype } & \multirow{2}{*}{$\frac{\text { Patients }}{\mathrm{n}(\%)}$} & \multicolumn{3}{|l|}{ PFS } & \multicolumn{3}{|l|}{ OS } \\
\hline & & & $\begin{array}{l}\text { Number of } \\
\text { events }(\%)^{a}\end{array}$ & $\begin{array}{l}\text { I 8-month } \\
\text { PFS rate (\%) }\end{array}$ & $P$-value & $\begin{array}{l}\text { Number of } \\
\text { events }(\%)^{a}\end{array}$ & $\begin{array}{l}\text { I8-month } \\
\text { OS rate (\%) }\end{array}$ & $P$-value \\
\hline \multirow{4}{*}{ FASL-844 C>T (rs763। I0) } & $\mathrm{CC}$ & $12(17.6)$ & $5(4 I .7)$ & 45.0 & & $5(4 I .7)$ & 45.0 & \\
\hline & CT & $32(47.1)$ & $28(87.5)$ & 5.0 & & $18(56.2)$ & 38.2 & \\
\hline & TT & $24(35.3)$ & $20(83.3)$ & 16.7 & & $10(41.7)$ & 58.3 & \\
\hline & $\mathrm{CT}+\mathrm{TT}$ & $56(82.4)$ & 48 (85.7) & 10.6 & $0.013^{c}$ & $28(50.0)$ & 47.5 & $0.60^{c}$ \\
\hline \multirow[t]{4}{*}{ FAS-670 A > G (rs | 800682) } & AA & $12(17.6)$ & II (9I.7) & 8.3 & & $8(66.7)$ & 27.8 & \\
\hline & AG & $33(48.5)$ & $23(69.7)$ & 23.0 & & $15(45.5)$ & 49.7 & \\
\hline & GG & $23(33.9)$ & $19(82.6)$ & 13.9 & & $10(43.5)$ & 54.8 & \\
\hline & $A G+G G$ & $56(82.4)$ & $42(75.0)$ & 19.1 & $0.44^{d}$ & $25(44.6)$ & 51.4 & $0.19^{d}$ \\
\hline
\end{tabular}

Notes: ${ }^{a}$ Row percentage. ${ }^{b}$ | 8 -month survival rate: cumulative proportion of survival derived from Kaplan-Meier analysis. ${ }^{c} P$-value was computed by log-rank test comparing FASL-844 CC genotype versus FASL-844 CT+TT genotypes. ${ }^{d P}$-value was computed by log-rank test comparing FAS-670 AA genotype versus FAS-670 AG+GG genotypes. Statistically significant $P$-values are shown in bold.

Abbreviations: OS, overall survival; PFS, progression-free survival.

investigated polymorphisms, the results show that the PFS rate at 18 months for FASL-844 CC genotype carriers was $45 \%$ versus $10.6 \%$ for FASL-844 CT/TT genotypes carriers (log-rank: 6.2; $P=0.013$ ) (Figure 1). On the other hand, no significant association was observed between PFS rate at 18 months and FAS-670 A $>$ G genotypes (log-rank: 0.6; $P=0.44$ ) (Table 2).

Of the clinicopathological and treatment variables, tumor stage (log-rank: $7.59 ; P=0.006$ ) and number of platinumbased cycles (log-rank: 78; $P \leq 0.001)$ were found as significant variables for PFS (Table 3). After adjusting for tumor stage and number of platinum-based cycles, FASL-844 C $>$ T polymorphism was still an independent predictor of PFS (HR $=3.77$ for FASL-844 CT/TT compared to FASL-844 CC; $P=0.012$ ) (Table 4).

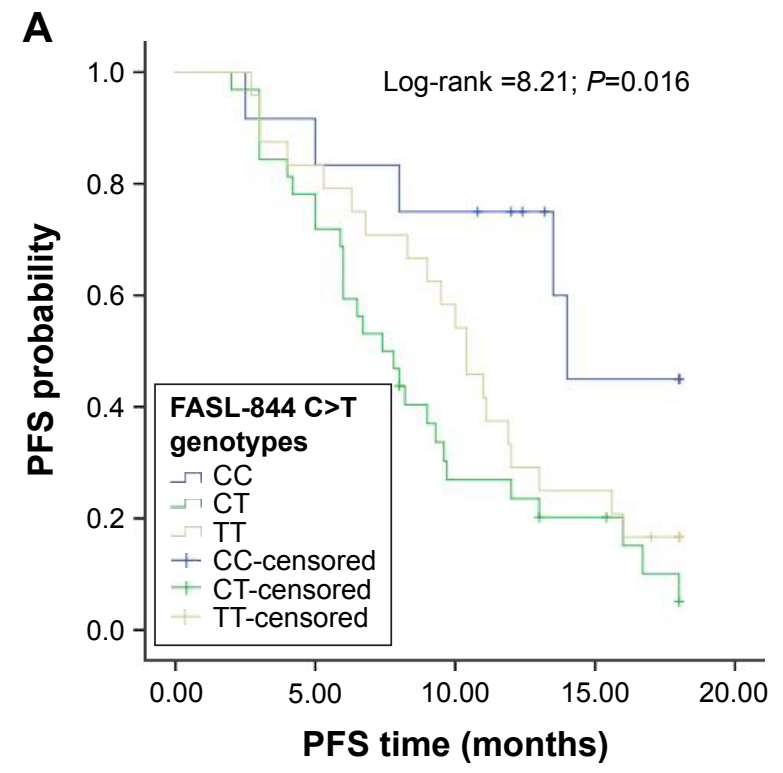

On studying OS, 33 patients (48.5\%) died, 22 patients (32.35\%) completed the follow-up period, while 13 patients $(19.15 \%)$ were lost to follow-up. The OS rate at 18 months for the entire cohort was $47.7 \%$. No significant association was observed between OS rate at 18 months and FASL-844 $\mathrm{C}>\mathrm{T}$ (log-rank: $0.27 ; P=0.60$ ) and FAS $670 \mathrm{~A}>\mathrm{G}$ polymorphisms (log-rank: 1.75; $P=0.19$ ) (Table 2). Number of platinum-based cycles was the only significant variable for OS (log-rank: 24.36; $P \leq 0.001$ ) (Table 3).

\section{Discussion}

Despite the rarity of MPM in the United States and other developed countries, MPM constitutes a major problem in Egypt because asbestos has not been banned until 2004. ${ }^{21}$ The majority of cases exists in Shobra El-Khaymah and

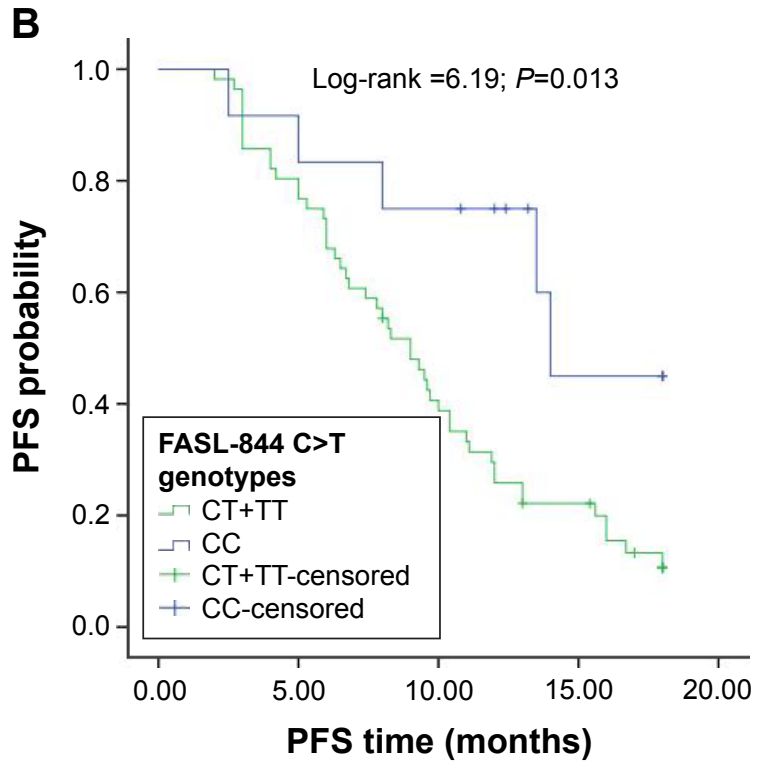

Figure I Kaplan-Meier curves of progression-free survival (PFS) for FASL-844 C>T (rs763I I0) genotypes (A) and FASL-844 CC versus FASL-844 CT/TT (B). 
Table 3 Influence of different clinical and treatment characteristics on survival assessed in univariate analysis in patients with malignant pleural mesothelioma treated with platinum-based chemotherapy $(n=68)$

\begin{tabular}{|c|c|c|c|c|c|c|}
\hline \multirow[t]{2}{*}{ Variable } & \multicolumn{3}{|l|}{ PFS } & \multicolumn{3}{|l|}{ OS } \\
\hline & $\begin{array}{l}\text { Number of } \\
\text { events (\%) }\end{array}$ & $\begin{array}{l}\text { I8-month } \\
\text { PFS rate (\%) }\end{array}$ & $P$-value ${ }^{c}$ & $\begin{array}{l}\text { Number of } \\
\text { events }(\%)^{a}\end{array}$ & $\begin{array}{l}\text { I8-month } \\
\text { OS rate (\%) }\end{array}$ & $P$-value ${ }^{c}$ \\
\hline \multicolumn{7}{|l|}{ Gender } \\
\hline Female & $21(84.0)$ & 12.0 & & II (44.0) & 52.8 & \\
\hline Male & $32(74.4)$ & 19.6 & 0.41 & $22(51.2)$ & 44.1 & 0.57 \\
\hline \multicolumn{7}{|l|}{$A g e^{d}$} \\
\hline$<55$ years & $21(65.6)$ & 28.8 & & $14(43.7)$ & 52.3 & \\
\hline$\geq 55$ years & $32(88.9)$ & 7.2 & 0.12 & $19(52.8)$ & 44.2 & 0.66 \\
\hline \multicolumn{7}{|l|}{ Smoking history } \\
\hline Nonsmoker & $24(85.7)$ & 10.0 & & $12(42.9)$ & 53.6 & \\
\hline Ever-smoker & $29(72.5)$ & 21.8 & 0.45 & $21(52.5)$ & 43.1 & 0.38 \\
\hline \multicolumn{7}{|l|}{ Asbestos exposure } \\
\hline Unexposed & II (9I.7) & 8.3 & & $7(58.3)$ & 38.9 & \\
\hline Exposed & $42(75.0)$ & 18.5 & 0.25 & $26(46.4)$ & 50.1 & 0.57 \\
\hline \multicolumn{7}{|l|}{ IMIG staging } \\
\hline ॥ & $13(59.1)$ & 34.4 & & $9(40.9)$ & 54.9 & \\
\hline III or IV & $40(87.0)$ & 8.2 & 0.006 & $24(52.2)$ & 44.1 & 0.27 \\
\hline \multicolumn{7}{|l|}{ Performance status } \\
\hline Zero & $8(80.0)$ & 0.0 & & $4(40.0)$ & 46.7 & \\
\hline One or more & $45(77.6)$ & 17.9 & 0.44 & $29(50.0)$ & 47.3 & 0.83 \\
\hline \multicolumn{7}{|l|}{ First-line regimen } \\
\hline Platinum/pemetrexed & $22(71.0)$ & 25.8 & & $16(51.6)$ & 42.0 & \\
\hline Platinum/gemcitabine & 31 (83.8) & 14.6 & 0.81 & I7 (45.9) & 52.2 & 0.47 \\
\hline \multicolumn{7}{|l|}{ Second line } \\
\hline No & & & & I5 (44.I) & 51.3 & \\
\hline Yes & & & & $18(52.9)$ & 44.9 & 0.93 \\
\hline \multicolumn{7}{|c|}{ Number of platinum cycles ${ }^{d}$} \\
\hline$<6$ cycles & $17(100)$ & 0.0 & & I5 (88.2) & 0.0 & \\
\hline$\geq 6$ cycles & $36(70.6)$ & 21.5 & $<\mathbf{0 . 0 0 1}$ & $18(35.3)$ & 60.2 & $<0.00$ I \\
\hline
\end{tabular}

Notes: ${ }^{\text {Row }}$ percentage. ${ }^{b} \mid 8$-month survival rate: cumulative proportion of survival derived from Kaplan-Meier analysis. ${ }^{c} S t a t i s t i c a l$ test: log-rank test, $P$-value $>0.05$ : nonsignificant. dMedian was used to categorize the variable. ${ }^{e}$ Environmental or occupational exposure to asbestos. Statistically significant $P$-values are shown in bold. Abbreviations: IMIG, International Mesothelioma Interest Group; OS, overall survival; PFS, progression-free survival.

Helwan, two suburbs of Cairo governorate, where asbestos factories were mainly localized. ${ }^{2}$ The data from the Department of Clinical Oncology and Nuclear Medicine, Ain Shams University, reported that at least 304 cases of MPM received therapy in the period between 2003 and $2008 .{ }^{21}$

Finding new predictive and prognostic markers in MPM field seems to be essential especially in the context of its reluctance against different treatment strategies. Thus, these new markers would help to distinguish the patients who would most likely benefit from these strategies and decrease the morbidities and mortalities associated with these choices. The objective of this study was to find new predictors to platinum-based regimens response in MPM patients. This is the first study to examine the role of FAS- $670 \mathrm{~A}>\mathrm{G}$ and FASL-844 C $>$ T polymorphisms in MPM setting.

Table 4 Influence of FASL-844 C>T and FAS-670 A > G polymorphisms on survival assessed in multivariate analysis in patients with malignant pleural mesothelioma treated with platinum-based chemotherapy $(n=68)$

\begin{tabular}{|c|c|c|c|c|c|}
\hline \multirow[t]{2}{*}{ Polymorphism } & \multirow[t]{2}{*}{ Genotype } & \multicolumn{2}{|l|}{ PFS $^{\mathbf{a}}$} & \multicolumn{2}{|l|}{ OS $^{\mathbf{b}}$} \\
\hline & & HR (95\% Cl) & $P$-value & HR (95\% Cl) & $P$-value \\
\hline \multirow[t]{2}{*}{ FASL-844 C>T (rs763II0) } & $\mathrm{CC}$ & Reference & & Reference & \\
\hline & $\mathrm{CT}+\mathrm{TT}$ & $3.77(1.34,10.62)$ & 0.012 & $1.37(0.52,3.60)$ & 0.52 \\
\hline \multirow[t]{2}{*}{ FAS-670 A>G (rs |800682) } & AA & Reference & & Reference & \\
\hline & $A G+G G$ & I.35 $(0.65,2.83)$ & 0.42 & $0.83(0.36,1.95)$ & 0.67 \\
\hline
\end{tabular}

Notes: a ${ }^{H R s}, 95 \% \mathrm{Cls}$, and $\mathrm{P}$-values were calculated by stratified Cox regression adjusted for stage of tumor and stratified by number of first-line chemotherapy cycles. ${ }^{b} \mathrm{HRs}$, $95 \% \mathrm{Cls}$, and $P$-values were calculated by stratified Cox regression stratified by number of first-line chemotherapy cycles. Statistically significant $P$-values are shown in bold. Abbreviations: $\mathrm{Cl}$, confidence interval; $\mathrm{HR}$, hazard ratio; OS, overall survival; PFS, progression-free survival. 
The results show that carriers of at least one T-allele of FASL-844 C $>$ T polymorphism have a higher risk for disease progression. This finding seems biologically plausible knowing that FASL-844 $\mathrm{C}>\mathrm{T}$ polymorphism is associated with alteration of FASL expression. ${ }^{14}$ Additionally, platinum agents exert their cytotoxic activity on MPM cells by the activation of FAS and FASL apoptosis system. ${ }^{10}$ Hence, regulation of FASL gene expression could be an important determinant of platinum chemosensitivity.

However, no statistically significant link was observed between this polymorphism and OS. This discrepancy in findings is clinically reasonable given the weak correlation between PFS and OS. ${ }^{22}$ Also, time restrain and withdrawal during follow-up period may lead to obscure the relationship between OS and FASL-844 C $>$ T polymorphism.

The current knowledge on the association between FASL-844 C $>$ T and clinical outcome in NSCLC is variable. Previously, Tao et al did not find any relationship between this polymorphism and PFS or OS in Chinese NSCLC patients. ${ }^{15}$ Also, these results were consistent with Park et al and $\mathrm{Wu}$ et al who did not observe a prognostic relevance for this polymorphism in Korean and Chinese NSCLC patients, respectively. ${ }^{17,23}$

Conversely, Sung et al demonstrated that this polymorphism could be used as a predictor of relapse and prognosis in a study included 385 Taiwanese patients diagnosed with NSCLC. ${ }^{16}$ Of note, Sung et al reported that carriers of two $\mathrm{C}$-allele were prone to higher risk of disease relapse and poorer survival. This finding seems contradictory to our finding which shows that carrying two $\mathrm{C}$-allele has a protective effect in terms of disease progression.

Interestingly, Mahfoudh et al showed that carriers of $\mathrm{C}$-allele are associated with better prognosis in Tunisian breast cancer patients who are similar to the population's ethnicity being from the North African region. ${ }^{18}$ Hence, it is hypothesized that the effect of FASL-844 C $>$ T on cancer progression or prognosis is affected by the studied ethnicity where our finding is consistent with study performed on North African population.

On the other hand, the results of this study show that there is no association between FAS-670 $\mathrm{A}>\mathrm{G}$ polymorphism and any of the clinical outcome. These findings are consistent with Wu et al but contradictory to Park et al and Tao et al who associated this polymorphism with survival in NSCLC patients. ${ }^{15,17,23}$ This discrepancy in the results could be attributed to the difference in the studied ethnicities or the difference in studied cancer or may be due to small sample size in our study which may obscure a significant relationship between this polymorphism and prognosis.
Finally the results did not show any association between the investigated polymorphisms and response rate which is consistent with the results shown by Tao et al. ${ }^{15}$ However, Liu et al demonstrated that FASL-844 C $>$ T polymorphism has an influence on NSCLC response to platinum agents. ${ }^{24}$ However, their conclusion is based on the interaction between this polymorphism and other genetic polymorphisms which is contrary to our study and Tao et al hypothesis where the effect of this polymorphism was studied solely and not as interaction with other genetic polymorphisms.

Despite the significant result obtained from this study, several limitations should be taken into consideration. Sample size was relatively small, which led to low statistical power. This could be justified by relatively low frequency of MPM and the prospective design of the study which are prohibitive factors to obtain large sample size.

Another potential limitation is the limited follow-up period of 18 months. However, this period is reasonable in the context of MPM where most patients die within 10-17 months after the onset of symptoms. ${ }^{25}$ Additionally, the 1- and 2-year survival rates in MPM Egyptian patients are estimated to be $60 \%$ and $27 \%$, respectively, which means that the survival rates would drop by more than half at 18 months. ${ }^{2}$ Lack of social support and low economic status of cases presenting to Department of Clinical Oncology and Nuclear Medicine, Ain Shams University, are factors that contributed to slightly high withdrawal rates from the study, reaching $\sim 19 \%$.

This cohort was heterogeneous regarding clinical characteristics and treatment protocols. This issue is overcome by studying the effect of each variable on the outcomes in univariate analyses, and multivariate analyses were performed to adjust for potential confounders.

The major strength point of this study is that the patients were prospectively followed up for PFS and OS where this overcomes the biases associated with retrospective design in studies that investigated these polymorphisms.

In conclusion, the results of this study demonstrate a role of FASL-844 $\mathrm{C}>\mathrm{T}$ polymorphism as a tumor progression predictor in MPM Egyptian patients treated with platinum-based therapy. This provides a novel insight into new predictive markers in MPM field. The conclusion should be validated by larger studies with uniformly treated patients.

\section{Acknowledgment}

The abstract of this paper will be presented as a poster at the IASLC (International Association for the Study of Lung Cancer) 17th World Conference on Lung Cancer, to be held 
from December 4 to December 7, 2016, in Vienna, Austria. The abstract will also be published in the Journal of Thoracic Oncology.

\section{Disclosure}

The authors report no conflicts of interest in this work.

\section{References}

1. Robinson BM. Malignant pleural mesothelioma: an epidemiological perspective. Ann Cardiothorac Surg. 2012;1(4):491-496.

2. Gaafar RM, Eldin NH. Epidemic of mesothelioma in Egypt. Lung Cancer. 2005;49(Suppl 1):S17-S20.

3. Ceresoli GL, Grosso F, Zucali PA, et al. Prognostic factors in elderly patients with malignant pleural mesothelioma: results of a multicenter survey. Br J Cancer. 2014;111(2):220-226.

4. Takuwa T, Hasegawa S. Current surgical strategies for malignant pleural mesothelioma. Surg Today. 2016;46(8):887-894.

5. Baas P, Fennell D, Kerr KM, Van Schil PE, Haas RL, Peters S. Malignant pleural mesothelioma: ESMO Clinical Practice Guidelines for diagnosis, treatment and follow-up. Ann Oncol. 2015;26(Suppl 5):v31-v39.

6. Vogelzang NJ, Rusthoven JJ, Symanowski J, et al. Phase III study of pemetrexed in combination with cisplatin versus cisplatin alone in patients with malignant pleural mesothelioma. J Clin Oncol. 2003; 21(14):2636-2644.

7. Nowak AK, Byrne MJ, Williamson R, et al. A multicentre phase II study of cisplatin and gemcitabine for malignant mesothelioma. Br J Cancer. 2002;87(5):491-496.

8. Ceresoli GL, Castagneto B, Zucali PA, et al. Pemetrexed plus carboplatin in elderly patients with malignant pleural mesothelioma: combined analysis of two phase II trials. Br J Cancer. 2008;99(1):51-56.

9. Itoh N, Yonehara S, Ishii A, et al. The polypeptide encoded by the cDNA for human cell surface antigen Fas can mediate apoptosis. Cell. 1991;66(2):233-243.

10. Stewart JHT, Nguyen DM, Chen GA, Schrump DS. Induction of apoptosis in malignant pleural mesothelioma cells by activation of the Fas (Apo-1/CD95) death-signal pathway. J Thorac Cardiovasc Surg. 2002;123(2):295-302.

11. Yang TY, Chang GC, Chen KC, et al. Pemetrexed induces both intrinsic and extrinsic apoptosis through ataxia telangiectasia mutated/ p53-dependent and -independent signaling pathways. Mol Carcinog. 2013;52(3):183-194.
12. Siena L, Pace E, Ferraro M, et al. Gemcitabine sensitizes lung cancer cells to Fas/FasL system-mediated killing. Immunology. 2014;141(2): 242-255.

13. Huang QR, Morris D, Manolios N. Identification and characterization of polymorphisms in the promoter region of the human Apo-1/Fas (CD95) gene. Mol Immunol. 1997;34(8):577-582.

14. Wu J, Metz C, Xu X, et al. A novel polymorphic CAAT/enhancerbinding protein beta element in the FasL gene promoter alters Fas ligand expression: a candidate background gene in African American systemic lupus erythematosus patients. J Immunol. 2003;170(1):132-138.

15. Tao KY, Li XX, Xu WZ, et al. Prognostic role of apoptosis-related gene functional variants in advanced non-small-cell lung cancer patients treated with first-line platinum-based chemotherapy. Onco Targets Ther. 2015;8:147-155.

16. Sung WW, Wang YC, Cheng YW, et al. A polymorphic $-844 \mathrm{~T} / \mathrm{C}$ in FasL promoter predicts survival and relapse in non-small cell lung cancer. Clin Cancer Res. 2011;17(18):5991-5999.

17. Park JY, Lee WK, Jung DK, et al. Polymorphisms in the FAS and FASL genes and survival of early stage non-small cell lung cancer. Clin Cancer Res. 2009;15(5):1794-1800.

18. Mahfoudh W, Bouaouina N, Gabbouj S, Chouchane L. FASL-844 T/C polymorphism: a biomarker of good prognosis of breast cancer in the Tunisian population. Hum Immunol. 2012;73(9):932-938.

19. Lima L, Ferreira JA, Tavares A, et al. FASL polymorphism is associated with response to bacillus Calmette-Guerin immunotherapy in bladder cancer. Urol Oncol. 2014;32(1):44.e41-44.e47.

20. Eisenhauer EA, Therasse P, Bogaerts J, et al. New response evaluation criteria in solid tumours: revised RECIST guideline (version 1.1). Eur $J$ Cancer. 2009;45(2):228-247.

21. Adel AM, Abdel Hafeez ZM, El Sheikh ET, El Sharawy IA, Gobran NS Malignant pleural mesothelioma: a retrospective analysis of clinicopathological and survival data. Thoracic Cancer. 2013;2(1):16-23.

22. Booth CM, Eisenhauer EA. Progression-free survival: meaningful or simply measurable? J Clin Oncol. 2012;30(10):1030-1033.

23. Wu X, Lu C, Ye Y, et al. Germline genetic variations in drug action pathways predict clinical outcomes in advanced lung cancer treated with platinum-based chemotherapy. Pharmacogenet Genomics. 2008;18(11):955-965.

24. Liu L, Wu J, Zhong R, et al. Multi-loci analysis reveals the importance of genetic variations in sensitivity of platinum-based chemotherapy in non-small-cell lung cancer. Mol Carcinog. 2013;52(12):923-931.

25. Zucali PA, Ceresoli GL, De Vincenzo F, et al. Advances in the biology of malignant pleural mesothelioma. Cancer Treat Rev. 2011;37(7): $543-558$.
OncoTargets and Therapy

\section{Publish your work in this journal}

OncoTargets and Therapy is an international, peer-reviewed, open access journal focusing on the pathological basis of all cancers, potential targets for therapy and treatment protocols employed to improve the management of cancer patients. The journal also focuses on the impact of management programs and new therapeutic agents and protocols on

\section{Dovepress}

patient perspectives such as quality of life, adherence and satisfaction. The manuscript management system is completely online and includes a very quick and fair peer-review system, which is all easy to use. Visit http://www.dovepress.com/testimonials.php to read real quotes from published authors. 